\title{
Multiple cardiovascular comorbidities and acute myocardial infarction: temporal trends (1990-2007) and impact on death rates at 30 days and I year
}

\author{
This article was published in the following Dove Press journal: \\ Clinical Epidemiology \\ 5 May 2012 \\ Number of times this article has been viewed
}

\author{
David D McManus ${ }^{1,2}$ \\ Hoa L Nguyen ${ }^{3,4}$ \\ Jane S Saczynski ${ }^{1,2}$ \\ Mayra Tisminetzky ${ }^{2}$ \\ Peter Bourell' \\ Robert J Goldberg ${ }^{2}$ \\ 'Department of Medicine, \\ ${ }^{2}$ Department of Quantitative Health \\ Sciences, University of Massachusetts \\ Medical School, Worcester, MA, \\ USA ${ }^{3}$ Institute of Population, Health \\ and Development, Hanoi, ${ }^{4}$ Oxford \\ University Clinical Research Unit, \\ Ho Chi Minh City, Vietnam
}

\begin{abstract}
Background: The objectives of this community-based study were to examine the overall and changing (1990-2007) frequency and impact on 30-day and 1-year death rates from multiple cardiovascular comorbidities in adults from a large central New England metropolitan area hospitalized with acute myocardial infarction (AMI).

Methods: The study population consisted of 9581 patients hospitalized with AMI at all 11 medical centers in the metropolitan area of Worcester, MA, during 10 annual periods between 1990 and 2007. The comorbidities examined included atrial fibrillation, diabetes, heart failure, hypertension, and stroke.

Results: Thirty-five percent of participants had a single diagnosed cardiovascular comorbidity, $25 \%$ had two, 12\% had three, and 5\% had four or more comorbidities. Between 1990 and 2007, the proportion of patients without any of these comorbidities decreased significantly, while the proportion of patients with multiple comorbidities increased significantly during the years under study. An increasing number of comorbidities was associated with higher 30-day and 1-year postadmission death rates in patients hospitalized with AMI.

Conclusion: Patients hospitalized with AMI carry a significant burden of comorbid cardiovascular disease that adversely impacts their 30-day and longer-term survival. Increased attention to the management of AMI patients with multiple cardiovascular comorbidities is warranted.
\end{abstract}

Keywords: acute myocardial infarction, comorbidities, survival, cardiovascular disease, trends, population-based

\section{Introduction}

Due in part to the aging of the US population, the number of individuals with multiple concurrent chronic diseases has increased significantly during the past several decades. ${ }^{1-4}$ The presence of multiple comorbid conditions makes clinical decisionmaking and patient management challenging, increases the risk for adverse outcomes, and is associated with higher medical care costs. ${ }^{5-9}$

Coronary artery disease disproportionately affects older individuals and is associated with a high burden of additional comorbidities. ${ }^{7,10}$ Although disease states such as diabetes, hypertension, heart failure, and atrial fibrillation share independent associations with increased mortality in patients with acute myocardial infarction (AMI) ${ }^{11-14}$ it is unclear whether these and other important comorbidities cluster together, or if comorbid disease burden is associated with variability in treatment or 
reduced survival. ${ }^{4,15,16}$ Moreover, data are lacking on how these disease patterns, and patient-related outcomes, may have changed during recent years.

The objectives of the present study were to examine nearly 2-decade-long trends (1990-2007) in the prevalence of six important chronic conditions, namely atrial fibrillation, diabetes, heart failure, hypertension, myocardial infarction, and stroke, singly and in combination, in 9581 patients hospitalized with AMI. These comorbid illnesses were selected for examination on the basis of established associations with the development of AMI or adverse AMIrelated prognosis and their relative frequency in this patient population. ${ }^{13,17,18}$ We examined the clinical correlates of multiple comorbid conditions, evaluated whether the presence of multiple concurrent chronic diseases influences hospital and posthospital admission mortality in patients hospitalized with AMI, and determined whether the prognosis associated with these chronic conditions has changed during the years under study. Data from the population-based Worcester Heart Attack Study were utilized for this investigation. ${ }^{19-23}$

\section{Methods}

The Worcester Heart Attack Study is an ongoing populationbased investigation that is examining long-term trends in the incidence, hospital, and postdischarge case-fatality rates of AMI among residents of the Worcester metropolitan area ( 2000 census estimate: 478,000 ) hospitalized at all 16 greater Worcester medical centers on an approximate biennial basis beginning in 1975. ${ }^{19-23}$ The periods under study were selected because of the availability of grant funding and for purposes of examining changes in our principal study outcomes over an approximate alternate yearly basis. Fewer hospitals $(n=11)$ have been included during recent study years due to hospital closures, mergers, and conversion to chronic care facilities. Of the current hospitals, three are considered to be tertiary care/university-based medical centers.

The details of this study have been described previously. ${ }^{19-23}$ In brief, computerized printouts for patients admitted to all greater Worcester hospitals with primary and/or secondary discharge diagnosis codes consistent with the presence of possible AMI were obtained, and International Classification of Disease (ICD) codes in which AMI may have been diagnosed were reviewed for purposes of sample selection (ICD-9 codes 410-414, 786.5). Cases of possible AMI treated at all greater Worcester medical centers were independently validated according to predefined criteria for AMI. ${ }^{21-23}$ This study was approved by the institutional review board at the University of Massachusetts Medical School.
Patients who satisfied the diagnostic criteria for AMI and were residents of the Worcester metropolitan area were included in this population-based investigation. Patients could have been included more than once in this investigation if they had been rehospitalized for AMI during the different years under study, with subsequent reclassification of the order of the present AMI (eg, initial versus prior). Patients who developed symptoms of AMI after hospital admission or after an interventional procedure or surgery were excluded because we wanted to study AMI that occurred de novo in the broader community setting.

\section{Data collection}

Information about demographic characteristics, comorbidities, and clinical data was abstracted from the hospital medical records of patients with confirmed AMI by trained study physicians and nurses. Information was collected about patient age, sex, race, marital status, body mass index, comorbidities, AMI type ( $Q$ wave versus non- $Q$ wave), hospital treatment practices, inhospital complications, and hospital discharge status. Discharged hospital patients were followed on an annual basis through review of records for additional hospitalizations and search of death certificates at state and local divisions of Vital Statistics. Some form of additional follow-up after being discharged from the hospital through either review of postdischarge medical records or death certificates was obtained for more than $99 \%$ of discharged hospital survivors from the cohorts included to date with follow-up completed through 2008.

The presence of prior atrial fibrillation, diabetes, heart failure, hypertension, and stroke was identified based on review of hospital medical records and physician's progress notes. These comorbidities were considered to be present if they had been previously diagnosed in hospitalized patients. Patients with missing data on previously diagnosed atrial fibrillation (2\%) were excluded from the analyses. There was no missing data on the other comorbidities examined. Multiple comorbidities were defined as the presence of one or more of these previously diagnosed comorbid conditions; further categorization into cutpoints of any two, any three, and at least four of these comorbidities was also carried out. In addition, common clusters of comorbidities among the six conditions examined were identified. For purposes of these latter analyses, a common cluster was defined as any combination of two or more comorbidities present in at least $2 \%$ of the study population. The primary outcomes of interest were 30-day post hospital admission total death rates and 1 -year all-cause mortality. 


\section{Data analysis}

The overall prevalence of multiple comorbidities, and changes over time therein, were calculated and compared using the Chi-square test for trends. In examining whether these trends were affected by other patient demographic and clinical characteristics, a multinomial logistic regression analysis was used to adjust for potential confounding factors, with patients without any of the comorbidities examined serving as the reference group. These factors were chosen based on findings from prior studies including age $(<65,65-74,75-84, \geq 85$ years), sex, race (white versus nonwhite), marital status (single, married, divorced, widowed), type of AMI (Q wave versus non-Q wave), and clinical complications that patients may have developed during their acute hospitalization (eg, heart failure, atrial fibrillation, cardiogenic shock). Study years were grouped into five periods (1990-1991, 1993-1995, 1997-1999, 2001-2003, and 2005-2007) for ease of analysis.

In examining the influence of multiple comorbid conditions on posthospital admission, all-cause death rates, age-sex adjusted survival curves for 30-day and 1-year mortality according to number of comorbidities were created. In addition, multivariable-adjusted Cox regression models yielded estimated hazard ratios and $95 \%$ confidence intervals for the risk of dying at selected time points according to the number of cardiovascular comorbidities that had been previously diagnosed (a list of controlling variables is included in the footnotes of the tables). Proportional hazard assumptions were checked graphically for all variables by plotting a Cox model versus Kaplan-Meier curves, and the assumptions of these models were satisfied (data not shown). Interaction terms between number of comorbidities present and study period were included in the multivariable-adjusted regression models for the purpose of examining whether any observed associations may have changed significantly over time. All analyses were performed using Stata software (version 11.0; Stata Corporation, College Station, TX).

\section{Results}

The study sample consisted of 9581 residents of the Worcester metropolitan area hospitalized with independently validated AMI at all greater Worcester medical centers in the 10 study years between 1990 and 2007. The average age of this patient population was 70 years, $57 \%$ were men, and the majority (93\%) were Caucasian.

\section{Prevalence of comorbidities}

Overall, a total of $7379(77 \%)$ patients had one or more of the comorbid conditions examined previously diagnosed at the time of their index hospitalization for AMI. In our study population, $35 \%$ of patients had a single comorbidity previously diagnosed, $25 \%$ had two, $12 \%$ had three, and $5 \%$ had four or more of these clinically important comorbid conditions present.

The number of comorbidities previously diagnosed in hospitalized patients was relatively stable between 1990 (mean 1.2, median 1) and 2007 (mean 1.6, median 1). However, significant changes were noted at the extremes of our study population distribution; the proportion of participants in whom no comorbid illnesses were present declined by nearly one-half ( $31 \%$ to $16 \%$ ), whereas the number of participants with four or more comorbidities diagnosed previously more than doubled ( $3 \%$ to $7 \%$ ) between 1990 and 2007 (Figure 1, $P$ for trend <0.05). These increasing trends remained statistically significant after adjusting for several potentially confounding factors in a multinomial logistic regression analysis (Figure 2). Compared with patients hospitalized during our earliest study years (1990-1991), patients hospitalized during the most recent periods under study (1997-1999, 2001-2003, and 2005-2007) were significantly more likely to report having multiple comorbidities present.

\section{Factors associated with multiple comorbidities}

Older individuals, women, nonwhite, and widowed patients were more likely to have multiple comorbidities previously diagnosed as compared with middle-aged persons, men, white, and other patients hospitalized with AMI (Table 1). Patients with a non-Q wave AMI and those who developed serious clinical complications of AMI, including heart failure and atrial fibrillation, were more often affected by multiple comorbidities as compared with respective comparison groups (Table 1).

\section{Comorbidity clustering}

In order to determine the frequency and prognostic impact of specific comorbidity patterns (or clusters), as well as changes over time therein, we examined the magnitude of all possible comorbidity clusters in our study population and how the presence of specific comorbidity combinations related to short- and long-term prognosis. As shown in Figure 3, the most common comorbidity cluster was hypertension and diabetes, which occurred in one out of every eight patients hospitalized with AMI. Hypertension, diabetes, and heart failure was the next most common comorbidity combination, affecting approximately $5 \%$ of study patients. Both of these comorbidity clusters, in addition to the combination of hypertension 


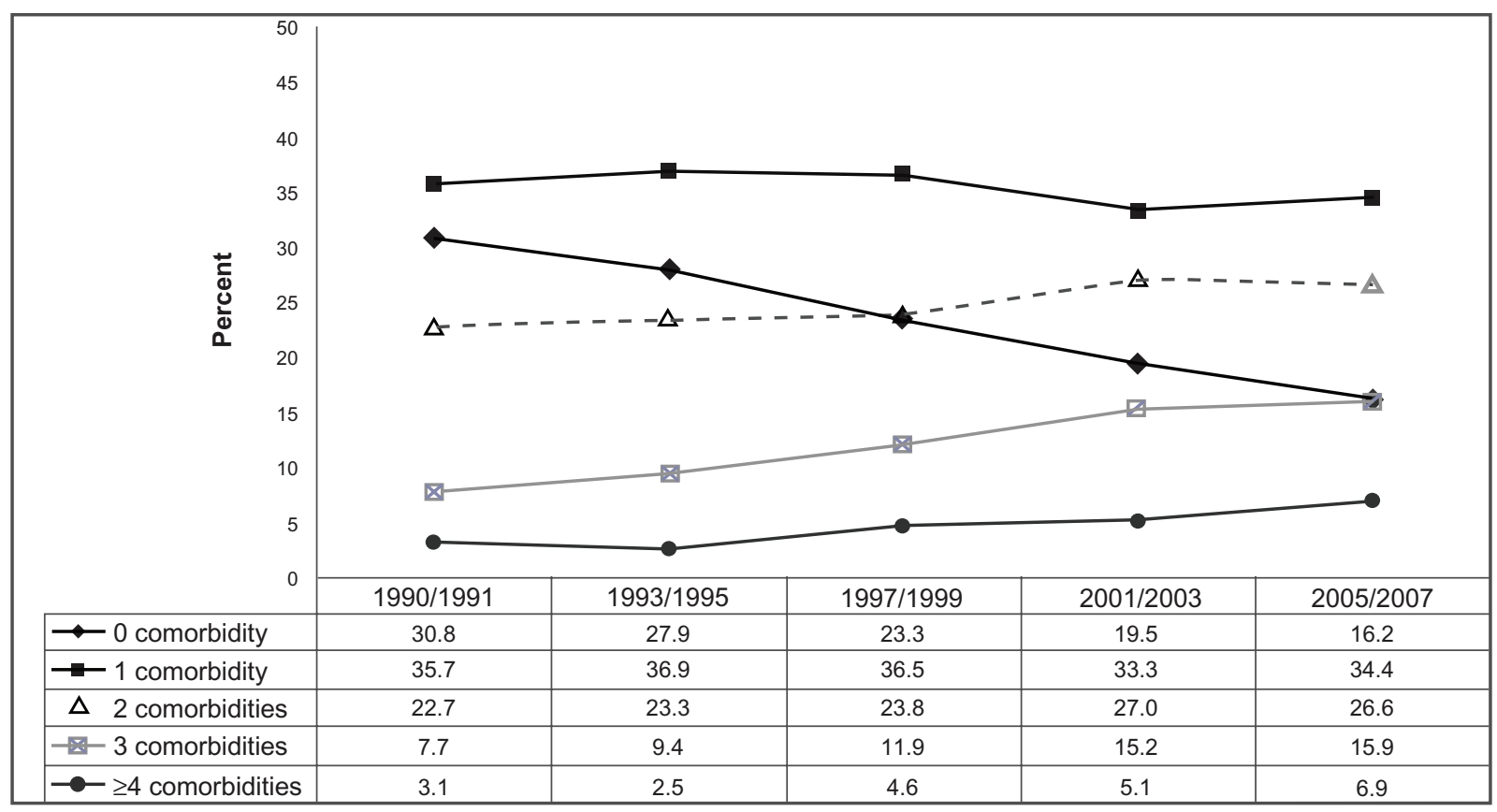

Figure I Prevalence of previously diagnosed multimorbidity among patients hospitalized with acute myocardial infarction according to study period.

and atrial fibrillation, increased in prevalence over the study period (Figure 3, $P$ for trend $<0.05$ ). These increasing trends remained statistically significant after adjusting for a number of potentially confounding demographic and clinical factors in a multinominal logistic regression model (data not shown).

\section{Mortality associated with multiple comorbidities and clusters of comorbidities}

In examining the relationship between number of comorbidities and all-cause mortality, having multiple comorbidities
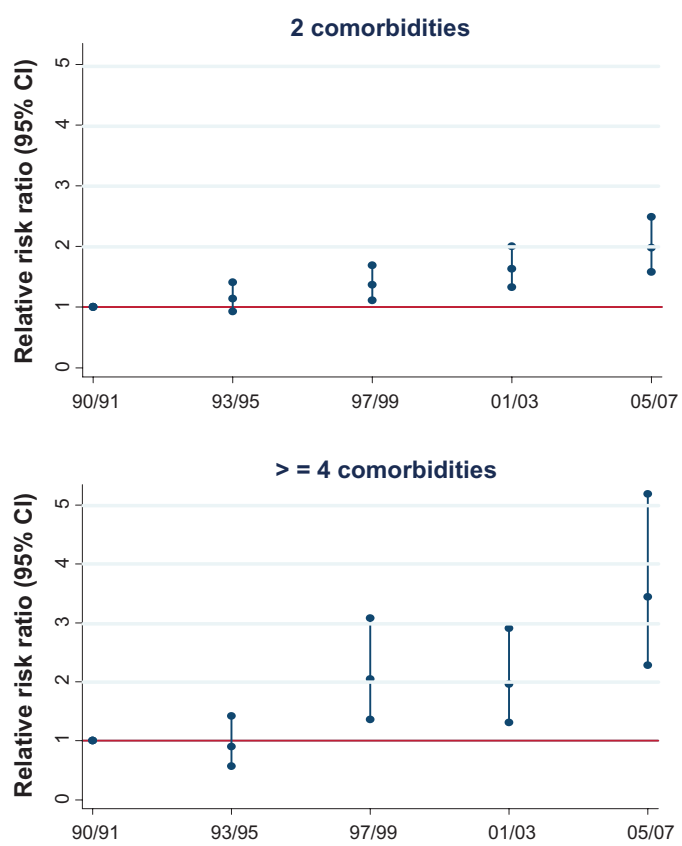

Figure 2 Changing trends of previously diagnosed cardiovascular comorbidities by study year.

Notes: Multinomial logistic regression model adjusted for age, sex, race, marital status, type of myocardial infarction (Q wave versus non-Q wave) and acute hospital complications (atrial fibrillation, heart failure, cardiogenic shock). Relative risk ratios $(95 \% \mathrm{Cl})$ calculated from multinominal logistic regression model and referent group includes patients without any comorbidity.

Abbreviation: $\mathrm{Cl}$, confidence interval. 
Table I Patient characteristics according to number of previously diagnosed cardiovascular comorbidities in patients hospitalized with acute myocardial infarction

\begin{tabular}{|c|c|c|c|c|c|c|}
\hline $\begin{array}{l}\text { Characteristic, } \\
\text { n (\%) }\end{array}$ & $\begin{array}{l}\text { No comorbidity } \\
(n=2202)\end{array}$ & $\begin{array}{l}\text { I } \\
(n=3377)\end{array}$ & $\begin{array}{l}2 \\
(n=238 I)\end{array}$ & $\begin{array}{l}3 \\
(n=1186)\end{array}$ & $\begin{array}{l}\geq 4 \\
(n=435)\end{array}$ & $P$ value \\
\hline \multicolumn{7}{|l|}{ Age group, years } \\
\hline$<65$ & I 202 (38.6) & II6I (37.3) & $541(17.4)$ & $|6|(5.2)$ & $47(1.5)$ & $<0.00$ I \\
\hline $65-74$ & $459(20.2)$ & $792(34.9)$ & $616(27.1)$ & $299(13.2)$ & $106(4.7)$ & \\
\hline $75-84$ & $377(14.0)$ & 921 (34.1) & $757(28.1)$ & $454(16.8)$ & $190(7.0)$ & \\
\hline$\geq 85$ & I59 (I I.4) & $461(33.0)$ & $438(3 \mid .3)$ & $255(18.2)$ & $86(6.2)$ & \\
\hline \multicolumn{7}{|l|}{ Sex } \\
\hline Men & |55| (28.4) & $1910(35.0)$ & $|22|(22.4)$ & $558(10.2)$ & $217(4.0)$ & $<0.00 \mathrm{I}$ \\
\hline Women & $651(16.0)$ & 1467 (35.6) & $1160(28.1)$ & $628(15.2)$ & $218(5.3)$ & \\
\hline \multicolumn{7}{|l|}{ Race } \\
\hline White & $2007(23.1)$ & 3101 (35.6) & $2155(24.7)$ & $1056(12.1)$ & $402(4.6)$ & 0.006 \\
\hline Nonwhite & $120(13.4)$ & $202(32.6)$ & I7| (27.6) & $100(16.2)$ & $26(4.2)$ & \\
\hline \multicolumn{7}{|l|}{ Marital status } \\
\hline Single & $282(23.4)$ & $392(35.3)$ & $279(25.1)$ & $136(12.2)$ & $23(2.1)$ & $<0.00$ I \\
\hline Married & I 402 (26.5) & $|88|(35.6)$ & $1243(23.5)$ & $54 I(10.2)$ & $224(4.2)$ & \\
\hline Divorced & $166(25.2)$ & $250(38.0)$ & 149 (22.6) & $66(10.0)$ & $27(4.1)$ & \\
\hline Widowed & $322(13.7)$ & 796 (33.9) & $660(28.1)$ & $416(17.7)$ & $|5|(6.4)$ & \\
\hline \multicolumn{7}{|l|}{ Type of AMI } \\
\hline $\mathrm{Q}$ wave & $983(33.6)$ & II 33 (38.7) & 577 (19.7) & $196(6.7)$ & $38(1.3)$ & $<0.00 \mathrm{I}$ \\
\hline Non-Q wave & $1210(18.4)$ & $2226(3.8)$ & $|78|(27.0)$ & $984(14.9)$ & $390(5.9)$ & \\
\hline \multicolumn{7}{|c|}{ Hospital complications } \\
\hline \multicolumn{7}{|l|}{ Atrial fibrillation } \\
\hline Yes & $250(14.5)$ & $522(30.3)$ & $492(28.6)$ & $311(18.1)$ & $148(8.6)$ & $<0.001$ \\
\hline No & $1952(24.8)$ & $2855(36.3)$ & $1889(24.0)$ & $875(11.1)$ & $287(3.7)$ & \\
\hline \multicolumn{7}{|l|}{ Heart failure } \\
\hline Yes & $486(13.2)$ & $1032(28.1)$ & II04 (30.1) & $756(20.6)$ & $295(8.0)$ & $<0.001$ \\
\hline No & $1716(29.1)$ & $2345(39.7)$ & $1277(2 \mid .6)$ & $430(7.3)$ & $140(2.4)$ & \\
\hline \multicolumn{7}{|l|}{ Cardiogenic shock } \\
\hline Yes & $121(20.2)$ & $213(35.6)$ & $155(25.9)$ & $87(14.6)$ & $22(3.7)$ & 0.208 \\
\hline No & $208 \mid(23.2)$ & $3164(35.2)$ & $2226(24.0)$ & $1099(12.2)$ & $4 \mid 3(4.6)$ & \\
\hline \multicolumn{7}{|l|}{ Study period } \\
\hline$|990-199|$ & $426(30.8)$ & $494(35.7)$ & $314(22.7)$ & $107(7.7)$ & $43(3.1)$ & $<0.001$ \\
\hline $1993-1995$ & $530(27.9)$ & 761 (36.9) & $444(23.3)$ & $179(9.4)$ & $48(2.5)$ & \\
\hline 1997-1999 & $485(23.3)$ & $76 I(36.5)$ & $495(23.8)$ & 247 (II.9) & $96(4.6)$ & \\
\hline $200 I-2003$ & $466(19.5)$ & $797(33.3)$ & $646(27.0)$ & $364(15.2)$ & $123(5.1)$ & \\
\hline $2005-2007$ & $295(26.3)$ & $624(34.4)$ & $482(26.6)$ & $289(15.9)$ & $125(6.9)$ & \\
\hline
\end{tabular}

Note: Comorbidities examined included atrial fibrillation, diabetes, heart failure, hypertension, and stroke.

Abbreviation: AMI, acute myocardial infarction.

previously diagnosed prior to hospitalization for AMI was significantly associated with poorer short- and long-term survival (Table 2, Figure 4). After adjustment for several potentially confounding demographic (eg, age, sex) and clinical factors (eg, hospital clinical complications) of prognostic importance, patients with two or more comorbidities were significantly more likely to have died within 30 days or at 1 year after hospital admission (Table 2). The risk of dying within 30 days and at 1 year after admission for AMI was directly related to the number of comorbidities present. The prognosis associated with having multiple comorbidities among patients hospitalized with AMI did not change significantly over time ( $P=0.57$ for interaction).
Patients with AMI and several of the most common comorbidity clusters were at higher risk for dying within 30 days and at 1 year after hospital admission, even after adjustment for several potentially confounding factors (Table 3 ). In particular, patients with the following combinations: hypertension + heart failure, hypertension + stroke, hypertension + diabetes + heart failure, or hypertension + diabetes + stroke, had greater odds of dying within 30 days or by 1 year after hospitalization for AMI than patients without these comorbid conditions.

\section{Discussion}

In this community-wide investigation of central Massachusetts residents hospitalized with an independently validated AMI 


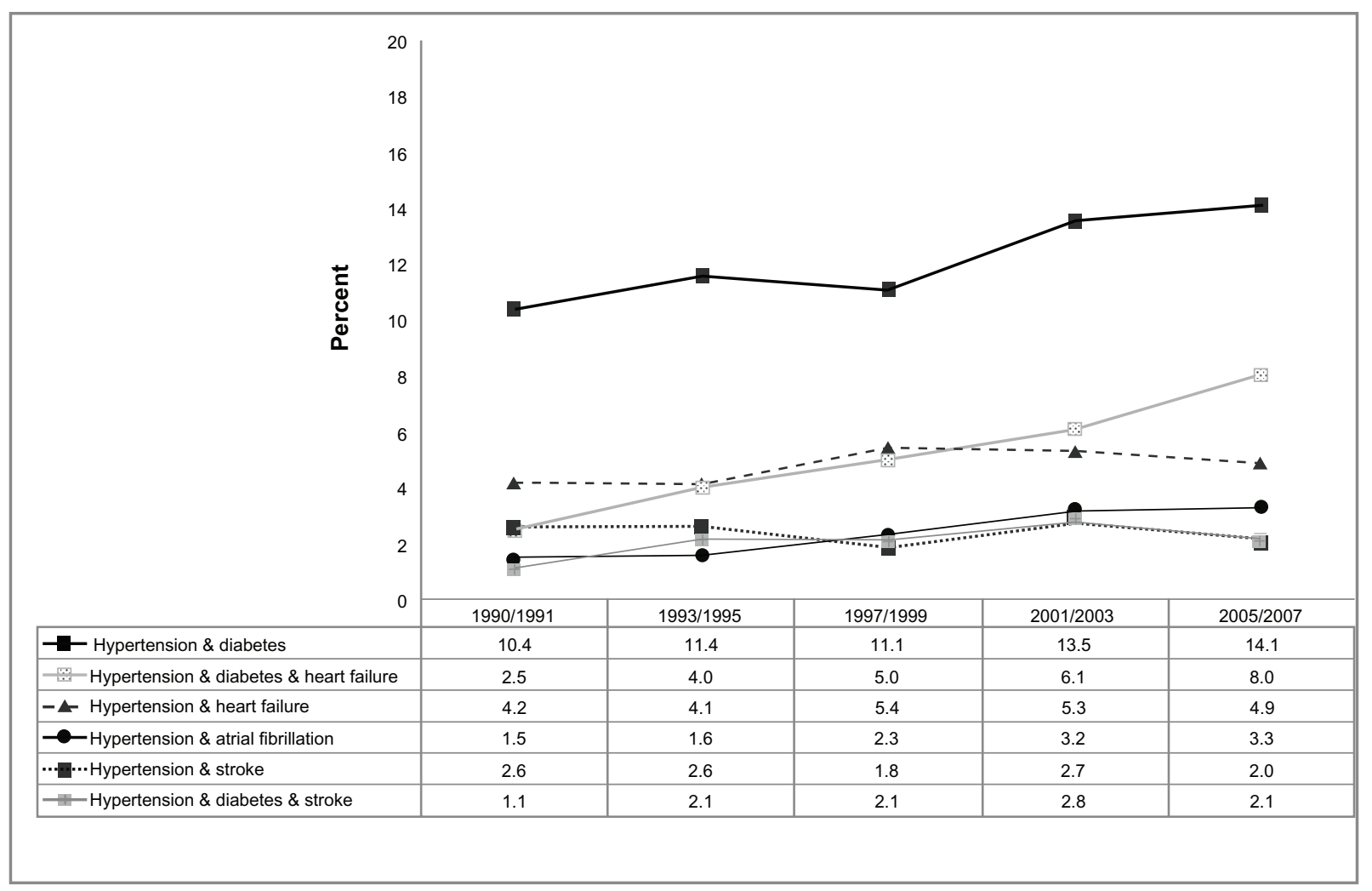

Figure 3 Proportion of patients with selected combinations of previously diagnosed cardiovascular comorbidities among patients hospitalized with acute myocardial infarction according to study period.

at all metropolitan Worcester medical centers between 1990 and 2007, the prevalence of previously diagnosed atrial fibrillation, heart failure, diabetes mellitus, and hypertension increased over time in hospitalized patients, as did the odds of being diagnosed with multiple comorbidities. Although the median number of comorbidities that were previously diagnosed in this patient population did not increase between 1990 and 2007, a dramatic increase in the percentage of hospitalized patients having four or more comorbid illnesses was observed, as was a concomitant decline in the proportion of patients without any of the comorbidities examined. The prevalence of several comorbidity combinations, most notably hypertension and diabetes, increased among AMI patients during the years under study. An increasing number of comorbid diseases was inversely associated with survival at 30 days and 1 year after admission to all central Massachusetts medical centers. In addition, despite increases in the proportion of patients with the highest burden of comorbidity, the adverse prognosis associated with having multiple comorbidities did not change significantly over the nearly 2-decade-long period under study.

Consistent with the published literature, we observed a high prevalence of the important cardiovascular comorbidities of atrial fibrillation, heart failure, prior MI, diabetes mellitus,

Table 2 Crude and multivariable-adjusted*30-day and I-year mortality according to number of previously diagnosed cardiovascular comorbidities in patients hospitalized with acute myocardial infarction

\begin{tabular}{|c|c|c|c|c|}
\hline \multirow{2}{*}{$\begin{array}{l}\text { Comorbidities } \\
\text { (n) }\end{array}$} & \multicolumn{2}{|c|}{ 30-day mortality } & \multicolumn{2}{|c|}{ I-year mortality } \\
\hline & Dead (n, \%) & Hazard ratio $(95 \% \mathrm{Cl})$ & Dead (n, \%) & Hazard ratio $(95 \% \mathrm{Cl})$ \\
\hline 0 & $198(9.01)$ & 1.00 & $330(15.02)$ & 1.00 \\
\hline I & $439(13.03)$ & $1.19(0.93-1.35)$ & $760(22.56)$ & $1.16(1.01-1.34)$ \\
\hline 2 & $422(17.76)$ & $1.49(1.23-1.80)$ & $816(34.34)$ & $1.62(|.4|-\mid .87)$ \\
\hline 3 & $252(21.32)$ & $1.64(1.32-2.03)$ & $532(45.01)$ & $1.94(1.66-2.26)$ \\
\hline$\geq 4$ & $97(22.30)$ & $\mathrm{I} .68(\mathrm{I} .28-2.2 \mathrm{I})$ & $233(53.56)$ & $2.31(1.91-2.78)$ \\
\hline
\end{tabular}

Notes: Comorbidities examined included atrial fibrillation, diabetes, heart failure, hypertension, and stroke. *Cox proportional hazards regression model adjusted for age, sex, race, marital status, study year, MI type (Q-wave versus non-Q wave) and acute hospital complications (atrial fibrillation, heart failure, cardiogenic shock). Abbreviations: $\mathrm{Cl}$, confidence interval; $\mathrm{Ml}$, myocardial infarction. 


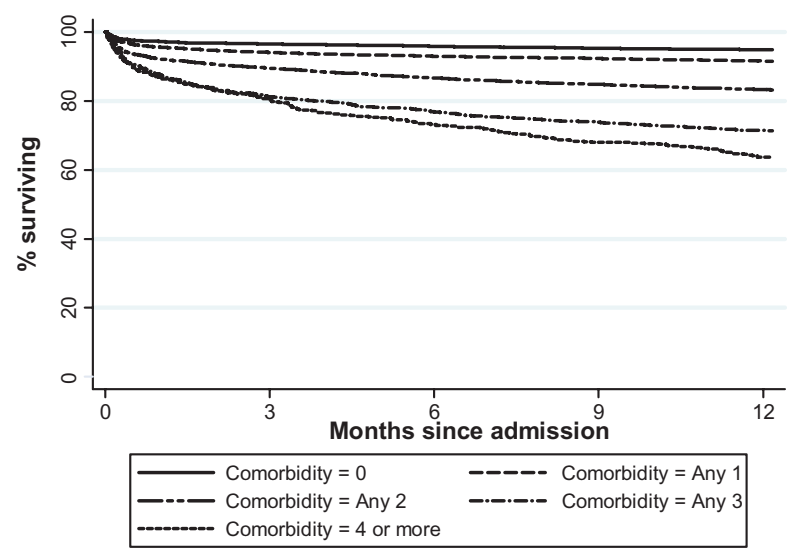

Figure 4 I-year age- and sex-adjusted survival rates among patients with acute myocardial infarction according to number of previously diagnosed cardiovascular comorbidities.

hypertension, and stroke in our patient population. ${ }^{13,24-26}$ The proportion of AMI patients affected by these comorbidities increased during the period under study, with the exception of prior MI and stroke. Our results confirm the findings of other contemporary investigations that have studied patients hospitalized with AMI, such as those from National Registry of Myocardial Infarction-2, which have also shown that the burden of atrial fibrillation, heart failure, diabetes mellitus, hypertension, and stroke have increased in this large multiregional population. ${ }^{10}$ Given the strong relationship between age, sex, and adiposity with the cardiovascular conditions under study, the observed trends are likely related to the increasing number of elderly, women, and obese patients hospitalized with AMI in our study population. 17,22,27

A major focus of the present investigation was to examine the magnitude of cardiometabolic comorbidities in patients hospitalized with AMI. The vast majority ( $>80 \%$ ) of patients in our study sample were affected by at least one of the comorbidities under study, and more than half were affected by two or more comorbid diseases; indeed, approximately one in every eight patients had four or more of the cardiovascular comorbidities examined. Although limited data on this topic exist, these observations validate our clinical impression that cardiovascular "multimorbidity" is the rule rather than the exception in patients hospitalized with AMI., ${ }^{4,15}$ Notably, rates of multimorbidity in our study were similar to, but slightly higher than, rates observed in similarly aged individuals included in prior investigations. ${ }^{7,8}$

There are several possible explanations for the high rates of multimorbidity observed in our hospitalized patient population. First, in light of the strong pathophysiological associations between diseases such as hypertension and heart failure, "causal comorbidity" has likely contributed to clustering of these diseases in our middle-aged and elderly patient population. "Complicating comorbidity," or the strong causal relationship between one disease and another, for example, between hypertension and stroke or obesity and diabetes, may also explain the frequent clustering of comorbidities in our study sample. ${ }^{28}$

The prevalence of patients without important cardiovascular comorbidities has decreased significantly, while the prevalence of patients with multiple comorbidities has increased significantly during the years under study in this population-based investigation. Reasons for the increasing rates of multimorbidity in our sample likely include enhanced risk factor and disease screening as well as improved patient and provider awareness of the importance of these chronic conditions between 1990 and 2007. ${ }^{17}$ However, the strong association between age, obesity, and several of the examined comorbidities, both singly and in combination, suggests that the increasing age and adiposity of patients hospitalized with AMI noted over time may also have contributed to observed trends. This hypothesis is supported by our observation that

Table 3 Crude and multivariable-adjusted 30-day and I-year mortality according to combination of previously diagnosed cardiovascular comorbidities

\begin{tabular}{|c|c|c|c|c|c|}
\hline \multirow[t]{2}{*}{ Comorbidity combination } & \multirow[t]{2}{*}{$\mathbf{n}$} & \multicolumn{2}{|c|}{ 30-day mortality } & \multicolumn{2}{|c|}{ I-year mortality } \\
\hline & & Dead (n, \%) & Hazard ratio $(95 \% \mathrm{Cl})$ & Dead (n, \%) & Hazard ratio $(95 \% \mathrm{Cl})$ \\
\hline No comorbidity & 202 & $19(9.0)$ & 1.00 & $330(15.0)$ & 1.00 \\
\hline Hypertension/diabetes & 1173 & $136(11.6)$ & $1.09(0.85-1.39)$ & $270(23.0)$ & $1.27(1.06-1.50)$ \\
\hline Hypertension/heart failure & 467 & $123(26.5)$ & $\mathrm{I} .82(\mathrm{I} .4 \mathrm{I}-2.34)$ & $239(5 \mathrm{I} .4)$ & $1.90(1.58-2.29)$ \\
\hline Hypertension/atrial fibrillation & 233 & $35(15.2)$ & I.I6 (0.79-I.7I) & $73(31.6)$ & $1.21(0.92-1.59)$ \\
\hline Hypertension/stroke & 224 & $47(21.0)$ & $1.73(1.21-2.47)$ & $84(37.5)$ & $1.76(1.36-2.28)$ \\
\hline Hypertension, diabetes, and heart failure & 507 & $92(18.2)$ & $1.40(1.06-1.85)$ & $214(42.3)$ & $1.79(1.48-2.16)$ \\
\hline Hypertension, diabetes, and stroke & 202 & $45(22.2)$ & $1.85(1.30-2.62)$ & $90(44.6)$ & $2.17(1.69-2.79)$ \\
\hline Other combinations & 4573 & $732(16.1)$ & 1.31 (1.10-1.57) & I37| (30.1) & I.43 (I.35-I.63) \\
\hline
\end{tabular}

Notes: Cox proportional hazard regression model adjusted for age, sex, race, marital status, study year, type of acute myocardial infarction ( $Q$ wave versus non- $Q$ wave) and acute hospital complications (atrial fibrillation, heart failure, cardiogenic shock). Comparisons are between patients with selected comorbidity clusters and patients without any comorbidity. 
the proportion of patients affected by specific comorbidity clusters that are strongly related to advancing age and obesity, such as hypertension and diabetes, increased most significantly over the study period. Similarly, because women and nonwhite patients with AMI were more likely to have multiple comorbidities, the increasing proportion of these patients in our community sample may have also contributed to increasing rates of multimorbidity noted during the years under study.

As has been reported previously, patients who reported having any of the cardiovascular comorbidities under study (with the exception of hypertension) were more likely to die during the first year after admission for AMI. We observed that having multiple comorbidities present was significantly associated with poorer survival at 30 days and 1 year after hospital admission for AMI and that the overall risk of dying increased linearly with the number of comorbidities present. These data suggest that the concomitant occurrence of multiple comorbid diseases exerts a more significant adverse effect on the short-term and long-term health of patients with an AMI than when they occur singly.,16 Our findings are supported by several prior investigations, including one involving more than 100,000 Medicare beneficiaries, which showed that the odds of clinical complications increased exponentially with increasing number of comorbidities (one comorbidity $=$ four complications per 1000 beneficiaries; four comorbidities $=34$ complications per 1000 beneficiaries $).{ }^{8}$

Our observation that multimorbidity is associated with poor short-term and better long-term survival is likely related to the fact that health care providers have little help in adjusting care for patients with multiple conditions, given that clinical trials and, in turn, practice guidelines, focus almost exclusively on patients with a single disease entity. Rates of complications from AMI treatment, such as bleeding, ${ }^{29}$ may be higher in patients with multiple comorbidities than in patients without any comorbid illness, due to factors such as drug-drug interactions or reduced drug clearance. Our results suggest that further population-based data on the use and impact of contemporary AMI treatments in patients with multiple comorbidities are needed.

\section{Study strengths and limitations}

The strengths of the present study include its communitybased design, its large sample of patients hospitalized with AMI, and its almost 2-decade-long perspective into changing trends in several clinically relevant endpoints. Our study limitations must also be recognized. First, although trends in the death rates of patients with AMI may have been influenced by cardiac treatments, our study did not examine the role and impact of hospital treatment practices, given the observational nature of this investigation, and our nonrandomized study design did not allow for adjustment for differences in these practices. Given that the majority of our study participants were white, our findings may lack generalizability to other ethnic or racial groups. Finally, physician thresholds for diagnosing several of the comorbid conditions under study may have changed over time, though it is unknown whether such a trend has occurred in our study population.

\section{Conclusion}

In this study of almost 10,000 patients hospitalized with AMI over a recent 2-decade period, we observed that the odds of having multiple cardiovascular comorbidities previously diagnosed in these high-risk patients increased significantly over time. Presence of multimorbidity was associated with reduced short- and long-term survival and the poor prognosis associated with multimorbidity persisted over the period under study. Our findings highlight the need for additional contemporary data to help guide the monitoring and treatment of patients with cardiovascular disease and multiple concurrent medical illnesses, as well as the importance of skilled and coordinated cardiovascular care for these highrisk patients.

\section{Acknowledgments}

We would like to express our gratitude to the physicians and nurses involved in the review of hospital medical records for this project. Grant support for this project was provided by the National Heart, Lung, and Blood Institute (RO1 HL35434 to RJG and 1U01HL105268-01 to RJG, DDM).

\section{Disclosure}

The authors report no conflicts of interest in this work.

\section{References}

1. From the Centers for Disease Control and Prevention. Public health and aging: trends in aging - United States and worldwide. JAMA. 2003;289: 1371-1373.

2. Parekh AK, Barton MB. The challenge of multiple comorbidity for the US health care system. JAMA. 2010;303:1303-1304.

3. Uijen AA, van de Lisdonk EH. Multimorbidity in primary care: prevalence and trend over the last 20 years. Eur J Gen Pract. 2008; 14 Suppl 1:28-32.

4. Vogeli C, Shields AE, Lee TA, et al. Multiple chronic conditions: prevalence, health consequences, and implications for quality, care management, and costs. J Gen Intern Med. 2007;22 Suppl 3:391-395.

5. Gijsen R, Hoeymans N, Schellevis FG, Ruwaard D, Satariano WA, van den Bos GA. Causes and consequences of comorbidity: a review. J Clin Epidemiol. 2001;54:661-674. 
6. Marengoni A, Rizzuto D, Wang HX, Winblad B, Fratiglioni L. Patterns of chronic multimorbidity in the elderly population. J Am Geriatr Soc. 2009;57:225-230

7. Sachdev M, Sun JL, Tsiatis AA, Nelson CL, Mark DB, Jollis JG. The prognostic importance of comorbidity for mortality in patients with stable coronary artery disease. J Am Coll Cardiol. 2004;43:576-582.

8. Wolff JL, Starfield B, Anderson G. Prevalence, expenditures, and complications of multiple chronic conditions in the elderly. Arch Intern Med. 2002;162:2269-2276.

9. Schäfer I, von Leitner EC, Schon G, et al. Multimorbidity patterns in the elderly: a new approach of disease clustering identifies complex interrelations between chronic conditions. PLoS One. 2010;5:e15941.

10. Rogers WJ, Frederick PD, Stoehr E, et al. Trends in presenting characteristics and hospital mortality among patients with ST elevation and non-ST elevation myocardial infarction in the National Registry of Myocardial Infarction from 1990 to 2006. Am Heart J. 2008;156: 1026-1034.

11. Califf RM, Pieper KS, Lee KL, et al. Prediction of 1-year survival after thrombolysis for acute myocardial infarction in the global utilization of streptokinase and TPA for occluded coronary arteries trial. Circulation. 2000;101:2231-2238.

12. Herlitz J, Karlson BW, Lindqvist J, Sjolin M. Prognosis during five years of follow-up among patients admitted to the emergency department with acute chest pain in relation to a history of hypertension. Blood Press 1998; 7:81-88.

13. Jabre P, Roger VL, Murad MH, et al. Mortality associated with atrial fibrillation in patients with myocardial infarction: a systematic review and meta-analysis. Circulation. 2011;123:1587-1593.

14. Norhammar A, Lindback J, Ryden L, Wallentin L, Stenestrand U. Improved but still high short- and long-term mortality rates after myocardial infarction in patients with diabetes mellitus: a time-trend report from the Swedish Register of Information and Knowledge about Swedish Heart Intensive Care Admission. Heart. 2007;93: 1577-1583.

15. Roe MT, Messenger JC, Weintraub WS, et al. Treatments, trends, and outcomes of acute myocardial infarction and percutaneous coronary intervention. J Am Coll Cardiol. 2010;56:254-263.

16. Ani C, Pan D, Martins D, Ovbiagele B. Age- and sex-specific in-hospital mortality after myocardial infarction in routine clinical practice. Cardiol Res Pract. 2010:752-765.

17. Dodd KS, Saczynski JS, Zhao Y, Goldberg RJ, Gurwitz JH. Exclusion of older adults and women from recent trials of acute coronary syndromes. $J$ Am Geriatr Soc. 2011;59:506-511.
18. McManus DD, Chinali M, Saczynski JS, et al. 30-year trends in heart failure in patients hospitalized with acute myocardial infarction. Am J Cardiol. 2011;107:353-359.

19. Goldberg RJ, Darling C, Joseph B, et al. Epidemiology of decompensated heart failure in a single community in the northeastern USA. Am J Cardiol. 2009;104:377-382.

20. Goldberg RJ, Gore JM, Alpert JS, Dalen JE. Recent changes in attack and survival rates of acute myocardial infarction (1975 through 1981). The Worcester Heart Attack Study. JAMA. 1986;255:2774-2779.

21. Goldberg RJ, Gore JM, Alpert JS, Dalen JE. Incidence and case fatality rates of acute myocardial infarction (1975-1984): the Worcester Heart Attack Study. Am Heart J. 1988;115:761-767.

22. Floyd KC, Yarzebski J, Spencer FA, et al. A 30-year perspective (1975-2005) into the changing landscape of patients hospitalized with initial acute myocardial infarction: Worcester Heart Attack Study. Circ Cardiovasc Qual Outcomes. 2009;2:88-95.

23. McManus DD, Gore J, Yarzebski J, Spencer F, Lessard D, Goldberg RJ. Recent trends in the incidence, treatment, and outcomes of patients with STEMI and NSTEMI. Am J Med. 2011;124:40-47.

24. Antman EM, Anbe DT, Armstrong PW, et al. ACC/AHA guidelines for the management of patients with ST-elevation myocardial infarction executive summary: a report of the American College of Cardiology/ American Heart Association Task Force on Practice Guidelines (Writing Committee to Revise the 1999 Guidelines for the Management of Patients With Acute Myocardial Infarction). Circulation. 2004;110: 588-636.

25. Iseki K, Kimura Y, Wakugami K, et al. Comparison of the effect of blood pressure on the development of stroke, acute myocardial infarction, and end-stage renal disease. Hypertens Res. 2000;23:143-149.

26. Rytter L, Troelsen S, Beck-Nielsen H. Prevalence and mortality of acute myocardial infarction in patients with diabetes. Diabetes Care. $1985 ; 8: 230-234$

27. Wu AH, Parsons L, Every NR, Bates ER. Hospital outcomes in patients presenting with congestive heart failure complicating acute myocardial infarction: a report from the Second National Registry of Myocardial Infarction (NRMI-2). J Am Coll Cardiol. 2002;40:1389-1394.

28. Eisenstein EL, Shaw LK, Nelson CL, Anstrom KJ, Hakim Z, Mark DB Obesity and long-term clinical and economic outcomes in coronary artery disease patients. Obes Res. 2002;10:83-91

29. Schellevis F, van der Velden J, van de Lisdonk E, van Eijk JT, van Weel C. Comorbidity of chronic diseases in general practice. J Clin Epidemiol. 1993;46:469-473.
Clinical Epidemiology

\section{Publish your work in this journal}

Clinical Epidemiology is an international, peer-reviewed, open access journal focusing on disease and drug epidemiology, identification of risk factors and screening procedures to develop optimal preventative initiatives and programs. Specific topics include: diagnosis, prognosis, treatment, screening, prevention, risk factor modification, systematic

\section{Dovepress}

reviews, risk \& safety of medical interventions, epidemiology \& biostatical methods, evaluation of guidelines, translational medicine, health policies \& economic evaluations. The manuscript management system is completely online and includes a very quick and fair peer-review system, which is all easy to use. 